\title{
Applying ASP to UML Model Validation
}

\author{
Mario Ornaghi, Camillo Fiorentini, Alberto Momigliano, and Francesco Pagano \\ Dipartimento di Scienze dell'Informazione, Università degli Studi di Milano, Italy \\ \{ornaghi, fiorenti, momiglia, pagano\}edsi.unimi.it
}

\begin{abstract}
We apply ASP to model validation in a CASE setting, where models are UML class diagrams and object diagrams are called "snapshots". We present the design and implementation of MSG, a snapshot generator for UML models that employs DLV-Complex as a generator engine, the answer sets representing the legal snapshots.
\end{abstract}

\section{Introduction}

The object of this research is the application of ASP to model validation in a CASE setting, in particular evaluating the "correctness" of formal specifications (or models) with respect to their requirements. Here, models are UML class diagrams [8] with constraints, typically written in OCL: a diagram should represent an abstraction of the problem domain; the objects populating a system state should represent a "snapshot" of a corresponding counterpart in the modeled world. In the UML, snapshots are represented by object diagrams. The legal snapshots are those satisfying the constraints that can be attached to the model to better specify the desired properties. In this context, tools for snapshot generation (SG) are an important part of the "weaponry" of light-weight formal methods. In fact, the relevance of SG for validation and testing in OO software development is widely acknowledged and a relevant part of the recently branded field of "Model-Based Testing" [4]. The latter ranges from model animation to ways of establishing partial certification such as model consistency and constraints independence checking.

This paper presents the design and implementation of a snapshot generator for UML models called "MSG" (read as "Message" and standing for "Milano Snapshot Generator", cooml.dsi.unimi.it/msg), which employs DLV-Complex [3] as a generator engine, the answer sets representing the legal snapshots. This is integrated in a system that takes as input any UML class diagram in XMI format and eventually displays back to the user the answers, i.e. the snapshots in the same format. The main theoretical contribution consists in a specialized representation of UML class diagrams into DLV-Complex, tailored to the fully automatic generation of non isomorphic snapshots The representation makes essential use of DLV-Complex's external functions, but still requires the introduction of an intermediate language (DLVExi) adding polymorphic types and existential quantification [5].

Background on UML. The Unified Modeling Language (UML) comprises a variety of model types for describing system properties, both static (e.g. class models, object models) and dynamic (e.g. state-machines, activity graphs). One of the more prominent

E. Erdem, F. Lin, and T. Schaub (Eds.): LPNMR 2009, LNCS 5753, pp. 457-463, 2009.

(c) Springer-Verlag Berlin Heidelberg 2009 
(a)

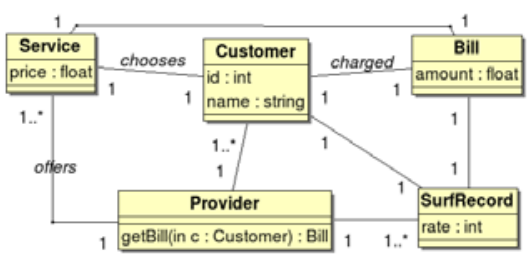

(b)

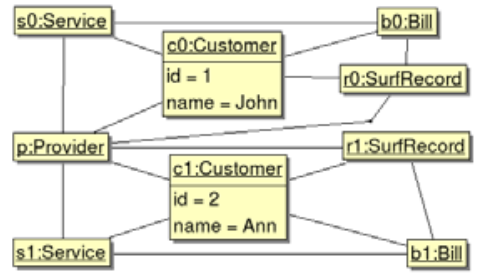

Fig. 1. A class diagram of an Internet Service Provider (a) and a snapshot for it (b)

model types is the class model (visualized as a class diagram) used to represent the underlying data model of a system in an object-oriented manner. A class diagram consists of a set of classes (rectangles) and relations among them, in particular associations (lines connecting rectangles), as shown in our running example (Fig. 11(a)), a simplified version of the ISP example from http: / /www. brucker. ch/projects/hol-ocl/ In this scenario a Provider offers some Service(s) at a certain price. A Customer chooses one of these services and she is charged a Bill according to her SurfRecord and the download rate. According to the UML "type-instance" dichotomy [8], classes, associations and class diagrams represent "types". Classes instantiate over "objects", associations over "links" and class diagrams over object diagrams, called "snapshots". Fig.1(b) shows a snapshot, where a provider $p$ (precisely, an object $p$ of class Provider) offers two services $\mathrm{s} 0, \mathrm{~s} 1$ and has two clients $\mathrm{c} 0, \mathrm{c} 1$, where $\mathrm{c} 0$ chooses $\mathrm{s} 0$, and so on. Association ends are decorated with various multiplicities. For example, the multiplicity $1 .{ }^{*}$ at the Service-end of the association offers denotes that a provider may offer one or more services, while 1 at the Provider-end indicates that a service is offered by one provider.

We remark that we can omit oids (object identifiers) in a snapshot. One way to look at this is considering oids as abstractions of memory addresses, which are transparent in OO programming. Thus, two snapshots of the same class diagram should be considered equal if they are isomorphic, where, roughly speaking, a snapshot isomorphism is a bijective map among oids that preserves the navigations. For example, if in the ISP snapshot we map c0 into $\mathrm{c} 1$ and $\mathrm{c} 1$ into $\mathrm{c} 0$, we get an isomorphic copy, i.e., morally, the "same" snapshot.

\section{Model Validation and Snapshot Generation}

Since requirements are informal, model validation can be only empirical, i.e., it is performed by comparing the formal model with the user's expectations. In this context, a snapshot generation tool (SGT) plays an important role. A SGT has two inputs: a model $M$ and a set $G$ of generation requests (GR), needed to make the number of snapshots finite. SGT output the set of legal snapshots that satisfy $G$. It allows us to perform various "experiments". To name one, the mere existence of a snapshot ensures that the model is consistent with its constraints.

Fig.2 2 shows the architecture and the data-flow of MSG. We start with the open source UML tool BOUML (http: / / bouml. free.fr/) to design diagrams and to generate the 
corresponding XMI representation. At the end of SG, BOUML displays the snapshots produced by the as $2 x m i$ module from the XMI format. The translation from XMI to DLV has been divided in two phases, using an intermediate language DLVExi. The latter allows us to decouple the representation of XMI models in logic from the definition and implementation of the generation request language. The Java component XMI2DLVEXI translates an XMI model $M$ into a DLVExi program $E_{M}$, which is a faithful representation of $M$ in the following sense: every legal snapshot of $M$ is represented by an "an-

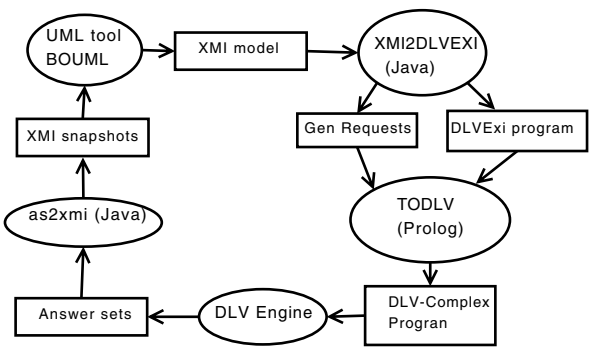

Fig. 2. The data flow view of the system swer set" of $E_{M}$ and every "answer set" of $E_{M}$ represents a legal snapshot of $M$. The component TODLV translates the program $E_{M}$ and the generation requirements $G$ into a DLV-Complex program $P_{M, G}$. The answer sets of $P_{M, G}$ are the answer sets of $E_{M}$ that satisfy $G$. DLV-Complex [2] is used as the generator engine.

\section{Representing UML into ASP}

In this section we discuss some encoding techniques for UML+OCL class diagrams in DLV-Complex. We do this via an intermediate language, DLVExi, which can be seen as an extension of DLV-Complex with a ML-like polymorphic type system and allowing conjunction and existential quantification in the head of clauses, as shown by the following fragment of trel, one of the DLVExi encodings of the UML with which we have experimented.

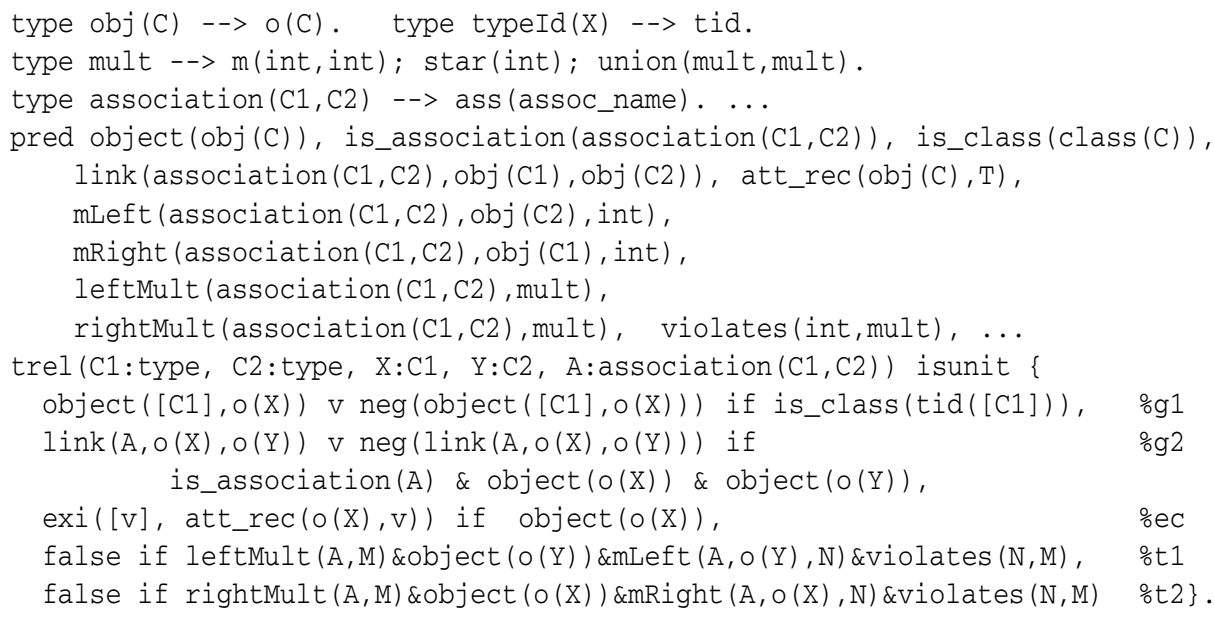

Before explaining trel, some brief comments on the DLVExi language are in order. Types are expressed analogously to datatype declarations in functional programming languages, where --> productions introduce polymorphic types by listing the 
type constructors (also called generators). For example, mult (multiplicity) is generated by $m$ (int, int), star(int) and, recursively, union(mult, mult). The ground mult-terms represent multiplicities, for example union $(\mathrm{m}(1,2)$, star (5)) represents $1 \ldots 2,5 \ldots{ }^{*}$. One use of (polymorphic) types is as wrappers, abstracting away from the types of the specific UML model. In particular, obj (C) is the type of the oids for a class type $\mathrm{C}$ and association $(\mathrm{C} 1, \mathrm{C} 2)$ the type of the associations between class $\mathrm{C} 1$ (left hand side) and C2 (right hand side). For the sake of type safe grounding [5], every ground term must have a unique type. To this aim, [5] introduces annotated functions $f_{J}(\ldots)$ and predicates $p_{J}(\ldots)$. In our concrete syntax, the annotations $J$ are enclosed between square brackets. For example, $t i d([\mathrm{C} 1])$ is the concrete syntax of $t i d_{C 1}$, while object $([\mathrm{C} 1], \mathrm{o}(\mathrm{X}))$ is object $C 1(\mathrm{O}(\mathrm{X}))$. Annotations may be left understood and are reconstructed by the system. If multiple annotations are possible, the system produces an error message. Polymorphic types allow us to decouple the general representation choices from the signature of the specific UML model. In this way, we can represent a UML model $M$ by a DLVExi theory $T_{M}=R \cup E_{M}$, where $R$ is a general "representation theory", and an "encoding theory" $E_{M}$ representing $M$ in $R$. The theory $R$ does not depend on $M$, but only on the representation choices and the generation strategy. For example, the above trel corresponds to a relational representation of the associations, by means of the predicates object ( 0 ) ("O is a live object") and $\operatorname{link}(A, 01,02)$ ("01 and 02 are linked by the association A"). According to the "guess and test" methodology of DLV [9], live objects and links are guessed by the rules $\% \mathrm{~g} 1$ and $\% \mathrm{~g} 2$, while $\% \mathrm{t}$ and $\%$ 2 "test" the multiplicity constraints. The XMI2DLVEXI component translates $M$ into the encoding theory $E_{M}$. For example, part of the ISP-encoding (Fig.1 (a)) is:

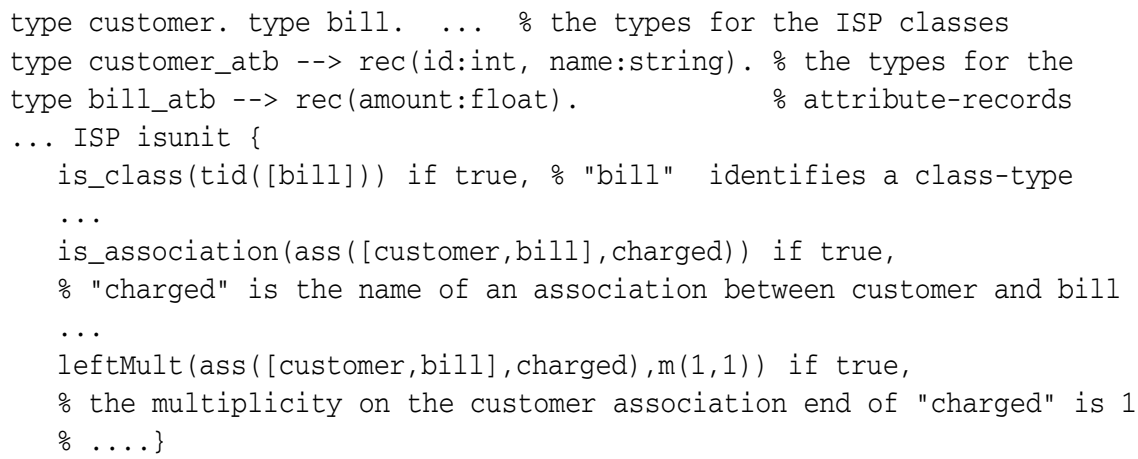

Inputs to the TODLV component are the theory $T_{M}=R \cup E_{M}$ and a set $G R$ of generation requests. The output is a DLV-Complex program. The generation requests suggest a finite set of possible object identifiers and a finite set of attribute values, in order to get finitely many models. Examples of $G R$ for customer and bill are:

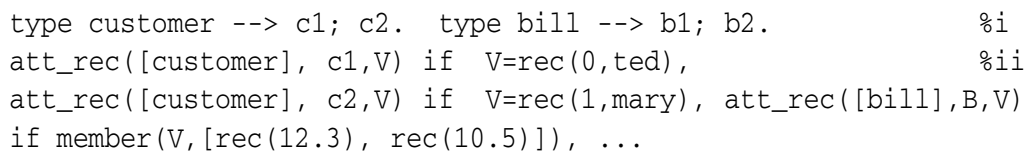

By $\% i$, we fix a finite set of possible oids, while $\%$ i gives a finite set of "witness-choices" for the existential variable $\mathrm{v}$ of the clause $\%$ ec. The TODLV component replaces the 
existential formula with a disjunction over the witness-choices, as shown in the clauses \% 4 of the following DLV-Complex program:

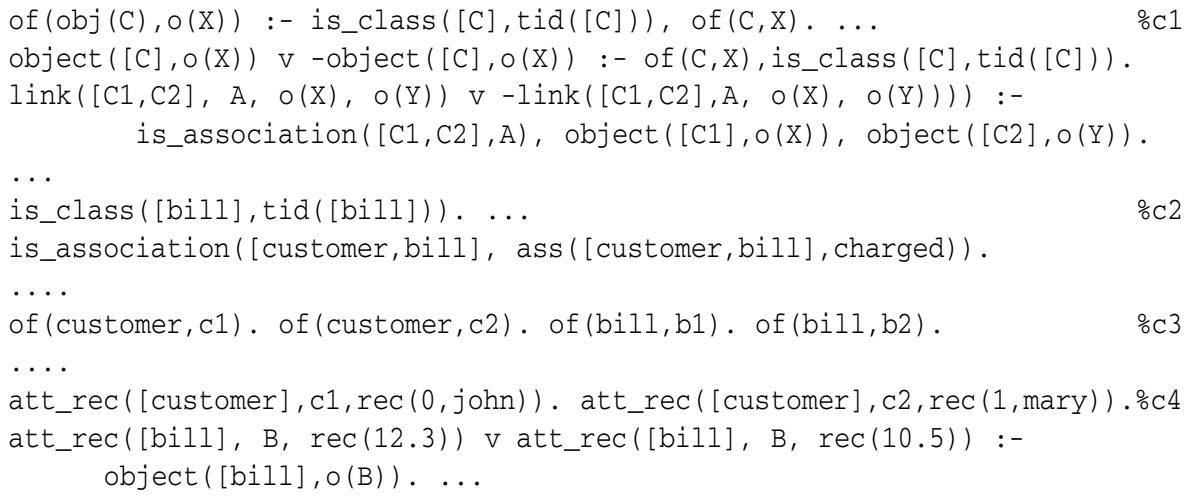

Clauses $\mathrm{C} 1$ are the DLV-Complex translation of trel (we use of $(C, X)$ as " $\mathrm{X}$ is of type C"), excluding $\%$ ec. Clauses c2 comes from the ISP-encoding, c3 from the generation requests for the oids and $c 4$ from the existential clause $\% e c$ and the related generation requests. We remark that TODLV does not perform grounding, which is left to DLV-Complex. Type and annotation reconstruction play a central role, since they enforce the correct grounding of polymorphic clauses. For example, the annotations of is_association clauses of $\mathrm{c} 2$ are used to instantiate the type-variables $\mathrm{C} 1, \mathrm{C} 2$ in the link-clause.

Finally we launch DLV-Complex with the above program, and we get back a set of stable models that represent the possible snapshots. Such models can be visualized as object diagrams using BOUML or a graphical tool. Here we show a snapshot that is consistent with the given specifications, yet does not fit with our expectations. This would suggest some problem in the modeling phase. Here, customer $c_{0}$ is associated with $b_{0}$, and $r_{1}$, while $c_{1}$ is associated with $b_{1}$ and $r_{0}$. This is surprising since, for instance, $r_{1}$ is the surfrecord of $c_{0}$, but $r_{1}$ and $c_{0}$ refer to different bills. This brings about very well the usefulness of lightweight formal methods and model validation in particular. One could do all sort of heavy functional verifica-

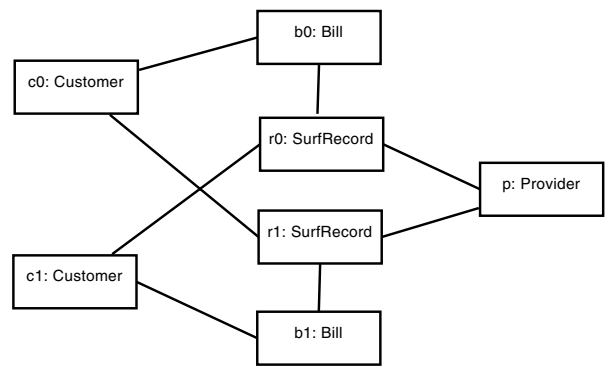
tion via interactive theorem proving only to discover that the initial model was under-specified and required further constraints. Without some clever notion of proof reuse, this would have meant a lot of wasted effort.

We conclude with a final comment concerning the intermediate language DLVExi. It has been introduced to enhance the expressive power of DLV-Complex, in order to define different representations of UML, of which trel is an example, minimizing the impact on the XMI2DLVEXI and TODLV components and on the GR language and its semantics. In particular, we have developed a functional representation tfun of 
the associations, not explained here for lack of space. The tfun representation drastically reduces the number of the generated isomorphic snapshots. For example, without generating the attributes, with 1 provider and at most 2 customers we have 100 snapshots with trel, while only 6 with tfun. The non isomorphic snapshots are 4 .

\section{Related and Future Work}

Animation tools for UML diagrams such as state-chart, activity etc. are a commercial enterprise. Among academics, the USE tool [6] claims to be the only one supporting automatic SG; differently from us, SG requires the user to write Pascal-like procedures in a dedicated language. The issue of isomorphic models does not seem to be addressed and the performances of USE are very sensitive to the order of objects and attribute assignments [1]. Other animation and validation tools support different languages. Alloy [7] is based on first-order relational logic. The Alloy Analyzer compiles a formula in the Alloy language into quantifier-free booleans and feed to a SAT solver. According to [1], the Alloy Analyzer is the leading system for generation of instances of invariants, animation of the execution of operations and checking of user-specified properties. However, Alloy is not formally object-oriented, nor does it support UML and OCL.

We have described the design and implementation of MSG, a tool using ASP for MBT in the context of model validation of UML+OCL class diagrams. While the system is not yet ready to be released our preliminary experiments have shown that it compares favourably with the functionalities and the statistics reported in [1] w.r.t. our main "competitor", USE. Our main theoretical contribution has been the introduction of an intermediate language and of a representation of UML class diagrams tailored to the fully automatic generation of non isomorphic snapshots.

Future work include engineering the implementation, but also improve the representation, especially w.r.t. cyclic structures: the functional encoding yields rational terms. Possible approaches are coinductive techniques or identifying isomorphic graphs via classes of equivalence w.r.t. oid and link names, using a nameless representation. We plan to integrate one of the available compilers for OCL and address validation of pre/post conditions of methods supporting both forward and backward animation.

\section{References}

1. Aydal, E.G., Utting, M., Woodcock, J.: A comparison of state-based modelling tools for model validation. In: Paige, R.F., Meyer, B. (eds.) TOOLS (46). LNBIP, vol. 11, pp. 278296. Springer, Heidelberg (2008)

2. Calimeri, F., Cozza, S., Ianni, G.: External sources of knowledge and value invention in logic programming. Ann. Math. Artif. Intell. 50(3-4), 333-361 (2007)

3. Calimeri, F., Cozza, S., Ianni, G., Leone, N.: DLV-Complex, http://www.mat.unical.it/dlv-complex

4. Dalal, S.R., et al.: Model-based testing in practice. In: ICSE 1999, pp. 285-294 (1999)

5. Fiorentini, C., Momigliano, A., Ornaghi, M.: Towards a type discipline for answer set programming. In: Berardi, S., Damiani, F., de Liguoro, U. (eds.) TYPES 2008 Post-Proceedings. LNCS, vol. 5497, pp. 117-135. Springer, Heidelberg (2009) 
6. Gogolla, M., Bohling, J., Richters, M.: Validating UML and OCL models in USE by automatic snapshot generation. Software and System Modeling 4(4), 386-398 (2005)

7. Jackson, D.: Software Abstractions: Logic, Language, and Analysis. MIT Press, Cambridge (2006)

8. Larman, C.: Applying UML and Patterns: An Introduction to Object-Oriented Analysis and Design and Iterative Development. Prentice Hall, Upper Saddle River (2004)

9. Leone, N., et al.: The DLV system for knowledge representation and reasoning. ACM TOCL 7(3), 499-562 (2006) 